\title{
Gestión de la diversidad en escuelas chilenas de frontera
}

\section{Raúl Antonio Bustos González,* Alfonso Díaz Aguad**}

Perfiles Latinoamericanos, 26(51)

2018 | pp. $123-148$

DOI: $10.18504 / \mathrm{pl} 2651-005-2018$

\section{Resumen}

Este artículo explora el modelo de gestión de la diversidad que se observa en las representaciones de las prácticas docentes y que manifestó un grupo de profesores de alumnos inmigrantes en Arica, ciudad de la frontera chilena con Perú y Bolivia. Utilizando un enfoque cualitativo y a través de entrevistas en profundidad y el análisis de contenido, se ha podido advertir la inexistencia de medidas inclusivas en el aula, lo que los docentes justifican con el argumento de que su deber es tratar por igual a todos los estudiantes, demostrando así una postura asimilacionista ante la diversidad.

\begin{abstract}
This article explores from a qualitative approach, the management model of the diversity present in the representations of teaching practices, expressed by teachers of immigrant pupils in the city of Arica (Chilean border with Peru and Bolivia). Through in-depth interviews and analysis of content, you can perceive the lack of inclusive measures in the classroom, which is justified by the teachers in regard to its duty to treat all students alike, demonstrating a position assimilationist toward diversity.
\end{abstract}

Palabras clave: inclusión, estudiantes migrantes, frontera, gestión de la diversidad, educación, profesores.

Keywords: Inclusion, migrant students, border, diversity management, education, teachers.

* Doctor en Educación por la Universidad Autónoma de Barcelona. Académico de la Facultad de Educación de la Universidad de Tarapacá | rbgonzalez@academicos.uta.cl

** Doctor en Historia por la Universidad Alcalá de Henares. Académico de la Facultad de Educación de la Universidad de Tarapacá | adiaz@uta.cl 


\section{Introducción}

- a racionalidad moderna intenta dar un orden al universo y se vincula a relaciones de poder expresadas en prácticas como las que se dan en las estructuras penales o médicas, y que encarnan una estructura de dominación y poder caracterizada por rituales disciplinarios. El fin último de este proceso es la producción histórica del hombre útil, uniforme y predecible y la marginación del componente que diverge (Sauquillo, 1987).

La naturaleza del mundo globalizado y su necesidad de integración entre países chocan con los mecanismos que establecen y mantienen un "orden" social, y que adjudican un estatus a los distintos componentes de una comunidad. En este sentido, el sólido crecimiento de la migración emergente desde hace más de tres décadas obliga a observar esta irrupción como un objeto de análisis académico, a lo menos y por sobre todo, como un objetivo de políticas públicas, dado que la migración no es un acto de mudanza definitiva de residencia, sino una forma de vida: la forma espacial de una nueva existencia social.

Las regiones fronterizas son zonas de circulación permanente de personas, más allá de lo que pueda controlar la política pública. Este movimiento dinámico se trasforma en una instalación definitiva que adquiere rasgos propios en lo político, lo económico, lo social y lo cultural (Imilán, Márquez \& Stefoni, 2015).

Entre las políticas públicas que más desafía esta nueva realidad, se encuentran las relacionadas con la educación, lo que se refleja en las dificultades que surgen en las prácticas diarias de los protagonistas de este ámbito: estudiantes, profesores y apoderados, ${ }^{1}$ entre otros.

Desde esta perspectiva, la construcción de un marco institucional para alcanzar grados más inclusivos de los inmigrantes en Chile (Hernández, 2011) requiere de avances tanto en lo económico y laboral, como en el educacional, promoviendo en este último una educación basada en la interculturalidad, capaz de marcar el camino hacia la tolerancia (Riquelme \& Alarcón, 2008; Alvites \& Jiménez, 2011).

En virtud de tal situación, resulta necesario recuperar los aportes de autoridades educativas, docentes, estudiantes y padres y madres, a fin de obtener una percepción más cercana de las necesidades locales para coordinarlas con las metas institucionales (Mendoza, 2014). Es así que, desde un enfoque cua-

1 En el sistema educativo chileno, el "apoderado" es un término genérico que designa al responsable personal de la educación del estudiante, en general los padres, o en determinadas circunstancias los abuelos o tutores. 
litativo, este artículo explora el modelo de gestión de la diversidad mostrado en las representaciones que los profesores de alumnos inmigrantes manifiestan de su rol y del proceso de inclusión en general que se realiza en las escuelas de Arica, ciudad fronteriza con Perú, y puerta de entrada de población migrante a Chile.

Para cumplir esta tarea se recurrió a un grupo focal de doce profesores que trabajan en contextos en los que hay estudiantes migrantes; posteriormente se aplicaron diez entrevistas en profundidad con un mismo número de profesores de alumnos migrantes. Esto permitió ahondar en la representación que los profesores señalan respecto de su labor como agente gestor de la diversidad.

\section{Marco teórico}

La diversidad es vista tradicionalmente como ajena al precepto moderno del Estado nación, esto es, el protagonista de la ficción de homogeneidad cultural, premisa básica de una "sociedad normalizada" conformada de grupos cuyos modos de vida se desarrollan en un sistema convenido de normas (Romero, 1976). El fin último de este proceso es la producción histórica del hombre útil, uniforme y predecible, para dar forma a un sistema de poder asociado con el acceso a los recursos y el control de los individuos (Sauquillo, 1987) y en el que se integra aquello que es idéntico al saber y se excluye lo que le es antagónico. Esta situación se expresa en las políticas sociales relativas a la vivienda, la educación o la salud, con las que se establece una relación de causalidad; es decir, que las limitaciones y carencias personales (grado de escolaridad, mano de obra no calificada, falta de disciplina) se transforman en los obstáculos que impiden el logro de una mejor calidad de vida (Morales \& Molina, 2003).

Hannoun (1992) propone una tipología de gestión de la diversidad que contempla tres modelos basados en una lógica secuencial en la que el último supera a su antecesor. Esta mirada nace de los cambios que, observados en un conglomerado social, son producto de la coexistencia de diferentes culturas en un mismo territorio (Castro, 2011). Se trata de los siguientes.

a) Modelo asimilacionista. Corresponde a la primera forma en que las sociedades afrontan la diversidad cultural y que consiste en interpretarla como un problema que debe corregirse. La integración a la vida social de los miembros de las culturas ajenas supone la internalización de la cultura mayoritaria y la renuncia a los patrones identitarios (Duschatzky \& Skliar, 2000). Para Hannoun, el producto es el monoculturalismo y el etnocentrismo. 
De acuerdo a esto, el asimilacionismo puede ser considerado el proyecto político de crear una nación homogénea.

b) Modelo multiculturalista. Que se fundamenta en la aceptación de las diferencias. No obstante, el multiculturalismo no garantiza la igualdad entre los diferentes grupos y por ello debe ser considerado como necesario, pero insuficiente, pues conlleva el peligro de promover la fragmentación social (Touraine, 2000).

c) Modelo interculturalista. Que plantea la necesidad de un doble salto epistemológico (Tarrow, 1990), pasando del conocimiento de la diversidad a su valoración (Schmelkes, 2009). Para Hannoun este modelo soluciona la potencial fragmentación social promovida por el multiculturalismo (1992), gracias a que origina síntesis culturales.

\section{Migración y educación}

Según Booth \& Ainscow (2002: p. 9), desde la perspectiva educativa, "la inclusión se concibe como un conjunto de procesos orientados a eliminar o minimizar las barreras que limitan el aprendizaje y la participación de todo el alumnado". En los últimos años, el término inclusión ha sustituido al de integración, ya que este último parte del supuesto de que es necesario "integrar" al alumno "diferente", concibiendo así la vulnerabilidad como problema de un grupo minoritario (Dovigo, 2014). Por eso actualmente se ha pasado del modelo de "escuelas especiales" al de "escuela para todos". ¿En qué momento se atraviesa la frontera de la integración a la inclusión? Para Dovigo (2014: p. 92):

El paradigma que incluye implícitamente la idea de la integración es la asimilación, basada en la adaptación del alumno vulnerable o en situación de riesgo a la organización de la escuela que está básicamente estructurada sobre la base de los alumnos "normales", y donde el diseño de alumnos "especiales" todavía juega un papel marginal o residual. Dentro de este paradigma, la integración se convierte en un proceso basado principalmente en diferentes estrategias para lograr que el estudiante sea lo más similar posible a los demás.

Colocar una supuesta "normalidad" como referencia de conducta conduce a negar las diferencias en favor de un ideal de uniformidad. El mismo Dovigo señala que, si se cuestionaran los límites de la "normalidad", podríamos apreciar la verdadera inclusión al interpretar la situación no como un problema del indi- 
viduo, sino como dificultades debidas al contexto educativo o a la interacción de los estudiantes con las personas e instituciones (Dovigo, 2014).

Los modelos presentados arriba reflejan un patrón de normalidad dado que establecen qué podría interpretarse como escuela, buen alumno y buen docente, y resiste a gran parte de los intentos de modificación (Giovine \& Martignoni, 2011). Las escuelas con modelo asimilacionista enfrentan la tensión de la pertenencia sin gestionar ningún cambio en sus procesos educativos, convencidas de que el estudiante es quien debe adaptarse al sistema escolar. Para la escuela interculturalista, en contraste, los cambios debieran considerar los elementos centrales de i) revisar la importancia de la igualdad entendida como los aspectos comunes dentro de la diversidad, y ii) superar el etnocentrismo (Jiménez, 2013). No obstante, el escenario se parece mucho al descrito por Dovigo (2014: p. 90):

De hecho, nos encontramos con una situación en la que, además de muchas experiencias positivas que se pueden identificar como "buenas prácticas" sólidamente establecidas en los centros educativos, se manifiesta también la persistencia de una serie de dificultades en relación con diferentes aspectos de la aplicación del proceso de inclusión en las escuelas. Éstas se refieren tanto a las dimensiones estructurales como a la formación de los maestros y el personal que participan en la ejecución de las intervenciones, y a la calidad de las mismas intervenciones, lo que pone de relieve una persistencia de las dificultades por parte del sistema escolar $[\ldots]$

Para Dovigo (2014), la mayor dificultad radicaría en la tendencia a categorizar, dado que esto resulta en enfoques reduccionistas, configurando lo que él llama enfoque biomédico (2014: p. 97): "Este enfoque tiende a enmarcar la situación del problema (así como problemas de aprendizaje o comportamiento) como una condición principalmente debida a factores biológicos y de los individuos". En oposición, Dovigo propone el modelo social que concibe el fracaso estudiantil como el producto de la interacción entre el sujeto y su contexto. Así, la cultura sería el origen de las reglas que facilitan o impiden el acceso a determinados grupos de personas, interpretando la diferencia como anormalidad.

Este hecho se manifiesta claramente cuando se identifica la actitud preponderante entre el profesorado enfrentado a dicha realidad: el desinterés por conocer el contexto social en el que desempeña su labor, y la creencia cómodamente arraigada en que la situación se "normalizará" de manera natural "con el tiempo" (Abajo \& Carrasco, 2004). Ante desigualdades educativas y sociales, los propios prejuicios son el punto de partida. Por eso debe rechazarse la visión generalizada que perpetúa la condición migrante, interpretándolo siempre como receptor de tratos diferenciales, sin haber diagnosticado su realidad (Gairín \& Iglesias, 2010). 
En el contexto educativo de la región en estudio, Pavez (2013) y Zapata (2011) dan cuenta de la trascendencia de la interculturalidad y formación del profesorado para la escuela diversa como desafío emergente.

\section{Estrategias de apoyo e intervención para la diversidad y colectivos migrantes}

Con frecuencia se asocia la atención a la diversidad con el "apoyo social", entendido este como la percepción que tiene una persona de ser objeto de estima y parte de una red (Taylor et al., 2004: p. 355). Hombrados \& Castro ahondan en el concepto de apoyo social identificando esferas o dimensiones de su ejecución:

tres han sido los tipos de apoyo que se han venido reflejando en la mayor parte de las clasificaciones: emocional, instrumental e informacional (Schaefer, Coyne \& Lazarus, 1981). El apoyo emocional representa el sentimiento personal de ser amado y la seguridad de poder confiar en alguien. El apoyo instrumental hace referencia a la posibilidad de poder disponer de ayuda directa, y el informacional consiste en la provisión de consejo o guía para ayudar a las personas a resolver sus problemas (Hombrados \& Castro, 2013: pp. 109-110).

Estos mismos autores muestran que la percepción del apoyo social variará dependiendo del grupo al que se pertenece, e incluso, la propia percepción de apoyo tendrá variantes dependiendo del grupo que lo entregue. Por ejemplo, los estudiantes señalan que el apoyo informacional de los profesores es muy valioso, lo que desplaza a un segundo lugar el apoyo emocional y a un tercero el instrumental, de parte de esos mismos docentes (Hombrados \& Castro, 2013).

\section{Estrategias institucionales}

Tolosana (2014: p. 25) señala que, en torno a la heterogeneidad de los colectivos vulnerables, "quizá el rasgo compartido sea el carácter involuntario de su situación, es decir, no son responsables de las causas que les hacen vulnerables." Si reconocemos esto, se debe tener presente que los miembros de los colectivos vulnerables —entre estos, los migrantes — no siempre son capaces de superar su situación sin un acompañamiento institucional que los ayude a equilibrar el contexto en el que se desarrolla su experiencia vital. Sobre ello, Gairín (2014) puntualiza que cualquier intervención institucional que promueva el cambio 
educativo precisa enfrentar el desafío de aspectos curriculares, organizativos, y de desarrollo profesional y comunitario. Mientras que Gómez (2005) divide las estrategias de adaptación académica en dos tipos:

a) Medidas ordinarias. Que corresponden a estrategias en la programación de aula con las que se asume que una tarea de la labor docente consiste en planificar actividades y contenidos que aborden los objetivos de aprendizaje desde la premisa de la diversidad.

b) Medidas extraordinarias. Que deben ejecutarse en virtud de grupos que, con las medidas ordinarias, no estarían en condiciones de alcanzar los mínimos exigidos; esto es lo que pasa con los planes de compensación educativa para estudiantes con desfase de uno o dos años de formación.

Para Chile, el mayor desafío se circunscribe a que las iniciativas de educación intercultural bilingüe se han particularizado a contextos indígenas y como medidas de reparación social, pero se han dejado fuera a los colectivos emergentes que provienen de las migraciones contemporáneas (Mondaca \& Gajardo, 2013).

\section{Contexto del estudio}

Arica y Parinacota se sitúan al norte chileno en la frontera con Bolivia y Perú. En esta región casi se ha duplicado el movimiento migratorio entre 2001 y 2008, el cual pasó, según la Policía de Investigaciones de Chile, de 2614653 a 4640658 personas. Y de acuerdo a los datos preliminares del Instituto $\mathrm{Na}-$ cional de Estadísticas (INE) (2012), en esa zona los inmigrantes eran 12 299, de los cuales 5996 provenían de Bolivia y 4821 de Perú, representando así un $5.8 \%$ de la población.

Un aspecto singular del fenómeno migratorio en Arica es que aquí se permite un libre tráfico entre Tacna y Arica para chilenos y peruanos; basta mostrar el documento de identidad y que el desplazamiento cumpla con la condición de una permanencia limitada a siete días en el territorio de destino. En la práctica, la migración internacional inicia al superar la frontera regional que separa Tarapacá de Arica y Parinacota en la parte chilena.

En esta zona las dinámicas fronterizas han sido permanentes, lo que ha consolidado la idea de interacción transfronteriza y transnacional. En este sentido, las relaciones entre Arica, Perú y Bolivia han sido fundamentales para tres sociedades civiles que con frecuencia han enfrentado coyunturas políticas, pero que han potenciado entre sí la movilidad y circulación, en una creciente integración regional. 
En ello también han jugado un rol fundamental las redes sociales y de parentesco entre dichas sociedades. Recordemos que Arica durante casi cincuenta años vivió la realidad de poseer ciudadanos peruanos y chilenos en un mismo territorio. Por eso abundan los familiares consanguíneos con distinta nacionalidad: se trataba de una frontera política pero no sociocultural.

No deja de ser relevante el reconocimiento de la tendencia generalizada de asociar identidad cultural con fidelidad política, lo que es muy parecido a la tesis de la seguridad nacional vigente en Chile durante la década de $1980 .{ }^{2}$ Más aún si se considera que la gran mayoría de estudiantes migrantes en Arica y Parinacota proceden de Perú y Bolivia, países que la representación colectiva nacional ha incorporado con el rótulo de "enemigos".

Asimismo, uno de los mitos más arraigados en la conciencia histórica de Chile es el de su "superioridad" respecto de sus vecinos. Huellas de lo que es Chile y "el otro" en la zona de estudio se evidencian en el concepto chilenización, que identifica modernidad y progreso con el Estado chileno, y que se corresponde con los prejuicios actuales ante el arribo de población migrante al norte del país (Valdivieso, 2007; González, 2002).

Acerca de que se requiere erigir una memoria histórica con la que se construyan ciudadanos interculturales desde la escuela, se ha explorado el posible replanteamiento de la Guerra del Pacífico desde una mirada coherente con ese objetivo (Mondaca, Rivera \& Aguirre, 2013). No obstante, los desfiles y el nacionalismo insertos desde las prácticas educativas de la educación preescolar muestran cómo hay un aparato ideológico que opera desde edades muy tempranas (Mondaca, Rivera \& Gajardo, 2014) en relación con la chilenización y la desperuanización de la zona en disputa, y que el tema nacionalista tiene profundas raíces en el norte de Chile (Mondaca, 2008; Mondaca \& Aguirre, 2011; Díaz et al., 2012; Mondaca, Gajardo \& Sánchez, 2015).

\section{Marco aplicado: diseño y justificación metodológica del estudio de campo}

La finalidad de la investigación es comprender la representación que de su rol y del proceso de inclusión en general tiene el profesor que trabaja con migrantes, en específico en Arica, ciudad chilena fronteriza con Perú y Bolivia. Por

2 Concepto que define diversas acciones de las Fuerzas Armadas (favorecidas desde Estados Unidos y en el contexto de la guerra fría) que buscaban garantizar el orden interno y combatir ideologías, organizaciones o movimientos que pudieran apoyar al comunismo, lo que en su momento legitimó la toma del poder y la violación sistemática de los derechos humanos. 
esto el objetivo del estudio de campo fue recoger los testimonios que releven dichas representaciones. Así, se ha optado por una perspectiva cualitativa de investigación para tener como punto de partida el paradigma de la complejidad debido a que este permitirá dar cuenta del dinamismo y la multiplicidad de variables que interactúan en el fenómeno (Morín, 1995).

La premisa base es que cada caso por analizar es una realidad única, por lo que su comprensión se aborda desde su propia lógica interna. El trabajo de campo se organizó desde procesos cuyo fin ha sido construir un conocimiento más allá de estas mismas situaciones para así comprenderlas junto a otras más que se les vinculan, en la medida en que comparten semejanzas. Se busca universales concretos que se han alcanzado por medio de un análisis profundo de los casos particulares y de la comparación entre estos (Jiménez, 2007). Es un análisis con la flexibilidad necesaria para dilucidar lo estudiado y mantener el equilibrio que exige la paradoja del estereotipo, el cual nace cuando se agravan dos de sus características intrínsecas: la generalización y la rigidez (Navas, Holgado \& Sánchez, 2009).

Por tales causas se ha construido una descripción contextualizada de la que gradualmente emerge nuestra base teórica (teoría fundamentada), misma que de modo inductivo provee de hipótesis que guían nuevas indagaciones, las cuales se formulan recursivamente durante el desarrollo de la investigación (Jiménez, 2013).

La técnicas utilizadas han sido los grupos focales y la entrevista en profundidad, mismas que ofrecen la doble aproximación del investigador y de los participantes (Caballero, 2001; Jackson, 2001; Poveda, 2003). Estas herramientas fueron elegidas por su eficacia para aprehender las experiencias del docente, pues permiten conocer el punto de vista desde el cual él estructura su relación con el estudiante migrante y, por otro lado, porque facilitan el conocimiento de acontecimientos que no percibe directamente el investigador y que se expresan en los conceptos del entrevistado (Martín-Crespo \& Salamanca, 2007).

En cuanto al tamaño de la muestra, el criterio empleado se determinó por las necesidades de información; por eso el muestreo se ha orientado con la saturación de datos (Martín-Crespo \& Salamanca, 2007). Se ha recurrido a una muestra pequeña no aleatoria porque importa la calidad del informante y la entrega de información de mayor profundidad. De este modo, y aceptando que el proceso de investigación es cíclico y abierto, se inició con un procedimiento de conveniencia (grupos focales) para luego identificar casos confirmantes y desafiantes que enriquecieran las conceptualizaciones iniciales (entrevistas en profundidad).

Las metodologías se implementaron de forma independiente, pero enfocadas hacia un mismo objeto de estudio con el propósito de obtener resultados 
convergentes y ahondar al mismo tiempo en las problemáticas inicialmente identificadas en el grupo focal.

Como los instrumentos se aplicaron en fases sucesivas, la obtención de datos fue simultánea con su análisis, lo que iba determinando el momento del cese de la recogida de información. La reducción de los datos se consiguió mediante la categorización. Esta siguió criterios temáticos, pues así da pauta a más variedad y, por ende, a más información valiosa. El cuadro 1 recoge el tipo de establecimiento y la cantidad de unidades educativas consideradas en este artículo.

\section{Cuadro 1. Cantidad y tipos de establecimientos considerados en el estudio}

\begin{tabular}{lcc}
\hline \multirow{2}{*}{ Tipo de establecimiento } & \multicolumn{2}{c}{ Unidad educativa } \\
\cline { 2 - 3 } & Educación básica & Educación media \\
\hline Municipal $^{*}$ & 4 & 3 \\
Particular subvencionado** $^{*}$ & 3 & 4
\end{tabular}

${ }^{*}$ Establecimiento ubicado en la comuna de Arica, y por ello urbano, que depende administrativamente de la Municipalidad. **Establecimiento particular que recibe aportes del Estado (financiamiento mixto) y ubicado en Arica.

Fuente: Elaboración propia.

Hecho lo anterior, y para comprender mejor el objeto de estudio, se codificaron los datos utilizando una opción abierta dado que permitía la emergencia de categorías ampliables o redefinibles de acuerdo a lo que la misma investigación iba arrojando.

De igual modo se empleó el análisis de contenido tanto por su eficacia para clasificar la información obtenida como porque da la oportunidad de incorporar la originalidad del investigador. En tanto que se procedió a la transcripción de las expresiones orales para que su manejo durante el análisis fuera el de "expresiones-objeto".

Como se puede ver, se optó por una codificación y análisis emergentes, siguiendo los planteamientos de la Grounded Theory (Glaser \& Strauss, 1967). Una elección inspirada en Vásquez \& Martínez (1996) y Woods (1987), quienes sugieren no forzar los datos, sino partir de los que el propio trabajo de campo entrega. El carácter interpretativo se empleó en las discusiones y reflexiones finales, justo lo que sigue en este artículo.

\section{Discusión}

Tanto en los grupos focales, como en las entrevistas con los profesores se identificó la representación que los docentes construyen de sus estudiantes migrantes, y en particular del rol que los docentes juegan en el proceso de inclusión 
en la estructura educativa de la sociedad de acogida. Enseguida se reseña la información que se obtuvo, organizada mediante las categorías de Resultados académicos, Estrategias de inclusión, Evaluación del proceso de inclusión y Políticas institucionales. Se ejemplifican algunas de nuestras aseveraciones citando testimonios que expresen las representaciones de los profesores considerados en el estudio. Estas voces han sido individualizadas por las iniciales de los establecimientos en que los docentes se desempeñan.

\section{Resultados académicos}

La casi totalidad de los docentes que trabajan con estudiantes migrantes señalan que estos no se diferencian mayormente de los estudiantes chilenos en rendimiento académico. Sin embargo, las opiniones que divergen indican que los primeros superan a los segundos. Los docentes atribuyen esta ventaja relativa a la actitud frente al estudio, a que hay contenidos ya tratados en el desarrollo escolar del estudiante migrante y a las condiciones de este último en su contexto de origen: "[...] el migrante por esencia aprovecha más la oportunidad, estudia más, tiene mejores promedios [...]" (CCRSH), "Inicialmente, en los cursos inferiores de la educación media, se siente cierta diferencia de contenidos y temáticas curriculares, que los alumnos han tratado o estudiado en el extranjero [...]" (LAlopp).

Asimismo, los estudiantes migrantes manifiestan ventajas en términos de contenidos curriculares ya adquiridos. Por ejemplo, los de origen peruano —el grupo mayoritario - tuvieron una enseñanza primaria en su país (la educación básica chilena) de seis años y no de ocho como en Chile. Esto causa que los contenidos de séptimo y octavo chilenos ya sean conocidos por los peruanos: "Los desfases... noooo i... para nada!, no se presentan en estos estudiantes. Algunos conocen el contenido, pero... con otras metodologías y estrategias" (ED14RR).

Por otro lado, se corroboró que hay estudiantes migrantes que vienen de grupos sociales con acceso a una educación que, en Chile, por una condición de vulnerabilidad relativa, no es posible: "Cognitivamente es distinta porque siempre va un paso adelante, yo no sé si sea por la formación que tuvo en su país, porque ella en su país estaba en el mejor colegio particular católico" (CCRSH).

Algunos docentes asocian el mayor interés de los estudiantes migrantes por los estudios a su condición como tal: "Si es un migrante es porque en su país no tenía oportunidades y acá en Chile hay oportunidades como becas, cursos, cosas así y ellos las aprovechan, son más respetuosos, cumplen más y son comprometidos con su enseñanza" (CCRSH). Otros lo explican por una necesidad de aceptación social "También puede ser por obtener un lugar dentro de su grupo, en el fondo, por ser reconocidos y aceptados" (ED14RR). 
En las excepciones en que los profesores califican el rendimiento de los estudiantes migrantes como inferior al de los nativos, aluden a dos factores: $i$ ) la introversión, lo cual impide que consulten abiertamente a los profesores: "no realizan muchas consultas al profesor frente a sus compañeros" (CAD); y ii) se comportan de acuerdo a su herencia de una educación tradicional centrada en contenidos y no en el desarrollo de habilidades:

el enfoque de las asignaturas, sobre todo de lenguaje, es hacia las habilidades y no hacia los contenidos, por lo tanto, les dificulta el hecho de que no les dé un contenido concreto muy extenso para estudiar. Se confía, siendo que las habilidades se desarrollan durante la clase y eso se evalúa en base a un contenido. Lo otro es que las evaluaciones de nuestro colegio, de mi área, miden habilidades por arrastre, $y$ van subiendo gradualmente de nivel (LBJNF).

No obstante, cuando los profesores enfrentan una situación como esta optan por considerar que todo se origina en un problema individual asociado a una situación socioeconómica: "influye el nivel socioeconómico, la familia, el lugar de origen además del sistema educativo de los niños" (ED4RI).

De igual modo, aunque los menos, hay docentes que indican que la condición de migrante determina las dificultades académicas: "Pienso que debe ser porque estos niños vienen con una carga emocional muy fuerte, hay niños que estuvieron años sin sus padres y recién ahora están por fin ellos o arrancando de la muerte." (LBJNF) "Nunca me he cuestionado eso, pero creo que es por la cultura diferente que tienen que enfrentar, otro idioma, otros sabores, otros olores" (ED17JJSM).

Otros profesores identifican las conductas y conocimientos previos de los estudiantes migrantes según su país de origen: "tuve alumnos provenientes de Haití y República Dominicana. En esos casos noté el desfase de contenidos y habilidades" (LBJNF).

Pero las dificultades de más relevancia que han advertido los profesores se hallan en las habilidades para comunicarse en el contexto de acogida: "hay varios conceptos o chilenismos que estudiantes migrantes, que no son cercanos a nuestra realidad cultural, no comprenden, lo que les presenta un problema a la hora de relacionarse con sus pares, o hasta para poder estudiar" (CPN).

\section{Estrategias de inclusión}

La situación de los alumnos migrantes no es tema de reflexión entre los docentes, porque atestiguan su exitosa adaptación. Y los pocos que reconocieron otorgarles 
un trato especial refirieron que es de tipo administrativo y el que se les ofrece en el aula: "el único trato distinto puede ser que uno se toma un tiempo mayor para explicarles algo, o lo hace más lento para que entienda el idioma, más que nada, porque los términos que ellos usan son distintos a los de nosotros, pero más allá de eso, en notas, en todo... es igual" (NAC). Y señalaba otro docente:

No, lo único es que el Ministerio te pide, mensualmente, es una lista que va, me parece (duda el profesor) a la Cancillería de alumnos migrantes. A fin de establecer un cierto control hay una ley que exige que ellos tengan su residencia legalizada, entonces hay algunos casos que el niño no tiene ningún documento en regla, pero, por la Convención de los derechos del niño, tú no le puedes negar educación a nadie. Ellos quedan con un rut provisorio, un rut 100 millones, entonces se le va pidiendo al apoderado que vaya regularizando quienes a veces se demoran más de un año (c12).

En este hecho, como en otros parecidos, para los profesores que trabajan con estudiantes migrantes, esta "igualdad" necesita de procedimientos normalizadores, como el obtener certificación nacional, aunque el número 100 millones ya discrimine por ser el código que encabeza los documentos de identidad para extranjeros residentes en Chile: "a todos los alumnos se les trata por igual. El único requisito es que cuenten con su cédula de identidad chilena” (CAD). Hay además iniciativas coyunturales que asumen cierta formalidad, tal vez sin mayor conciencia de pasar como estrategias motivacionales:

hacemos actividades específicas cuando hay actividades deportivas; te cito el mundial de fútbol, ocasión que se le permite al alumno migrante venga con la polera de su país, para que ellos recuerden que tienen que valorar su origen. Porque hay algunos que reniegan, y algunas veces puede ser muy traumático. Pero después se dan cuenta que Chile es un país tranquilo, el país que andaban buscando. Pero como te digo, se les hace valorar su origen y que el resto valore el origen de ellos, por eso lo del Mundial. [...] Entonces venían todos con su camiseta de Chile, pero si tú eras de origen boliviano, colombiano, se podía venir con las camisetas de esos países. Aunque hubo varios de ellos que usaron la camiseta de Chile, entonces se les respeta, pero ellos toman su decisión. (Profesor de E12).

Otros docentes ocupan la experiencia vital y circunstancias propias de los estudiantes migrantes a manera de contenido motivacional: "Motivarlos a que se esfuercen más, ya que si sus padres vinieron hasta este país, era para darles a ellos un futuro mejor" (c12). Una acción que se acerca a la violencia simbólica, debido a que al niño se le recuerda permanentemente la condición migrante, 
se piensa que en esta se encuentran los determinantes de su conducta y se usa la culpa como estrategia.

En cuanto a las estrategias de control emocional, los profesores responsabilizan de esta función al personal de apoyo: "Por parte del psicólogo, para tratar el cambio repentino de cultura." (ED-14RR). "Es el deber de orientación: velar por la integridad personal y emocional de los estudiantes, previo informe del profesor jefe" (LA-1OPP).

El apoyo que recibe el estudiante migrante no aparece como una acción emergente de la tarea del profesor, sino como un producto de su comportamiento el cual determina si es o no merecedor de ello: "Tenemos cierto grado de diferencia hacia ellos, en la medida de que ellos se los ganen" (LA-1OPP).

Aunque, de acuerdo a los docentes, hay estudiantes migrantes que exigen un trabajo más personalizado: "son jóvenes igual a cualquier otro y hay que afrontarlos con nuevas formas pedagógicas, como clases que sean más personalizadas, entendiendo que las diferencias culturales que pueden haber en el aula son importantes" (CAD).

\section{Evaluación del proceso de inclusión}

En cuanto al nivel de inclusión de los estudiantes migrantes, la mayor parte de los profesores declararon ser testigos de un proceso exitoso, sobre todo por la condición fronteriza de Arica y la consecuente habitualidad del contacto con niños peruanos y bolivianos: "Hay que entender que Arica es una ciudad con muchos migrantes y cerca de dos países vecinos, a los cuales les debemos muchas formas culturales que hoy se expresan abiertamente. Por eso Arica, en cosas de integración está más avanzado, según mi percepción, con respecto a los migrantes" (CPN).

Mientras que en cuanto a su escolaridad, predominan los docentes que destacan la disposición de los estudiantes migrantes: "integrar a los niños migrantes no se hace complicado y da buenos resultados, ya que ellos también ponen de su parte" (CM). Un interés que para los docentes representa el principal indicador de inclusión puesto que hay un buen rendimiento académico y ausencia de problemas disciplinares graves. Aun así, la visión de algunos profesores es más crítica del proceso de inclusión y en consecuencia su punto de vista discrepa:

A ellos les cuesta integrarse. Prácticamente no se integran, lo que sí, conviven. La verdad creo que no hay esfuerzos por integrarlos, concretos, se dice cosas, de cómo debe ser el trato, pero no hay una estrategia fija, por ejemplo, cuando se pasa la Guerra del Pacífico no se pasan las dos visiones y esa sería una forma de integrarlos, 
cosas así del 21 de mayo. Uno como profesor trata de integrar las dos visiones por una cuestión personal, pero no es parte del currículo, entonces el currículo no da instancias de integración. El día lunes, por ejemplo, a pesar de tener un 37\% de alumnos extranjeros, se canta un solo himno nacional y bueno, yo lo entiendo, pero hay experiencias en Santiago en donde los alumnos migrantes superan el 50\% se canta el himno nacional de ambos países [...] (Profesor de CCRSH).

\section{Relación de los alumnos migrantes con sus pares chilenos}

En lo referente a las relaciones cotidianas entre estudiantes migrantes y nativos, la totalidad de los docentes entrevistados las consideran normales. Con todo, muchas de las conductas que los profesores catalogan como normales están lejos de ser deseables: "La relación entre ellos es normal, en el sentido que no hay sucesos significativos de discriminación. En el chileno existe la tendencia a hablar mal del inmigrante sin medir quién está al lado de nosotros, pero es una cuestión cultural del chileno, tratar de paisano, de indio, es una cuestión arraigada que está en el lenguaje. Eso es como normal, es una cuestión cultural que viene desde la casa” (CCRSH).

$\mathrm{Y}$ cuando los profesores reconocen que hay dificultades en las relaciones mencionadas, indican que los conflictos bélicos que enfrentó a Chile con los países vecinos son los núcleos de las diferencias. Acerca de esto, ellos argumentan que las familias son los agentes que han transmitido discursos centrados en la alteridad entre las naciones involucradas, omitiendo el rol que en ello ha jugado la educación formal: "La discriminación es más una cuestión adquirida y el tema se pone en el tapete cuando se habla de la Guerra del Pacífico, 7 de junio, etc. Sí, los niños chilenos son bastante discriminadores, pero es una cuestión inculcada desde la casa, porque en el aula se olvidan, en la sala son todos iguales, pero eso sí, tienen un discurso aprendido, desde la casa, desde la sociedad misma" (CCRSH).

Si bien los docentes afirmaron que estos problemas de convivencia aparecen fundamentalmente por las características individuales de los estudiantes, lo que deja fuera del análisis los factores de acogida:

En general, muy bien, normal digamos. Cuando hay alumnos... por ejemplo, el de quinto tiene problemas conductuales, pero con todos y ahí viene "el colombiano", "que este hizo eso", y él se escuda en esa situación. Pero los demás no tienen ese problema, entonces por eso te digo, va una situación específica de cada una de las personas. Pero en general, el de octavo es muy querido por sus compañeros. La niña que es boliviana también es muy querida por las compañeras. El que 
tiene problemas es él [el colombiano de quinto], pero yo creo tendría los mismos problemas en Colombia, es su carácter (c12).

Pero, en términos generales, los profesores observan que la relación entre alumnos migrantes y nativos es muy buena en el colegio; muchas veces por las cualidades de los primeros en cuanto a su trato y aplicación académica: "En los tres casos que conozco, los alumnos son queridos por todos sus compañeros [...] los alumnos de aquí, les piden los cuadernos a ellos, muchas veces" (NAC).

\section{Relación con la familia de alumnos migrantes}

La representación que los docentes declaran acerca del apoderado migrante es la de un padre y madre preocupados: "A ver, los apoderados son responsables, respetuosos, asisten a las reuniones regularmente y no presentan mayor problema" (цтрснме). Aunque, la inasistencia ocasional de estos a las actividades se interpreta como desinterés. Es probable que esta lectura insinúe una visión despectiva y de desconfianza del profesor ante el apoderado migrante:

Ahí hay alguna pequeña diferencia: el apoderado de un alumno migrante no responde como un apoderado "normal". Usan esa excusa que no sabían, que "no sé", cuando yo creo que en cualquier colegio, en cualquier parte de Sudamérica, o de los países vecinos, se trabaja igual acá. Entonces tú necesitas que estén presentes, no son constantes en las reuniones de apoderados, no son constantes en las citaciones... o estarán tan agobiados por tantos problemas [lo expresa con algo de decepción]. Yo creo que el adulto [migrante] está viviendo tantos problemas que no le alcanza el tiempo para la escuela, esa sería una explicación, pero no son tan presentes como los otros padres (ED-17JJSM).

Se conserva la misma mirada de superioridad, e incluso racista, entre el apoderado migrante y el "normal" en el siguiente testimonio:

Yo creo que el papá de un nińo migrante siente vergüenza. A través de mi experiencia con el trato con ellos yo lo he visto, participan, pero dentro del marco de la obligación, ellos no participan voluntariamente de las actividades, porque yo creo que sienten vergüenza. Hay papás de niños inmigrantes que tienen baja escolaridad, entonces no responden encuestas porque yo creo que no saben leer ni escribir, no porque no quieran, es difícil diferenciar entre el papá de un niño migrante, y el papá de un chileno andino, porque hay niños andinos que uno tiende a confundir, pero son niños andinos (CCRSH). 
Una apreciación que no es generalizada, pues la mayor parte de los docentes percibe diferencias entre los apoderados nacionales y los migrantes, en especial en cuanto a la preocupación por la situación escolar de sus hijos, esto se evidencia en otra respuesta: "La relación con los padres migrantes es buena, pero no es constante, ya que trabajan jornadas de varias horas. En cambio los padres chilenos, también es buena, pero no aceptan críticas de sus hijos o hijas, o en el otro extremo, que no se interesa en informarse sobre la situación de sus pupilos" (ED-17JJSM).

Si bien, y aunque se destaquen los elementos positivos de los apoderados migrantes, el relato de dichas experiencias adquiere ciertos tintes despectivos: "Hay apoderados locales que no están ni ahí, en cambio los 'peruanitos' no faltan a las reuniones" (EEE-5). El "peruanitos" deja entrever la representación de inferioridad ya referida arriba para otros aspectos de la relación con estudiantes migrantes.

\section{Diferencia entre los migrantes "tradicionales" y los "nuevos migrantes"}

De acuerdo a lo relevado entre los profesores que trabajan en contextos con presencia de estudiantes migrantes, se constata que ellos distinguen entre estudiantes migrantes de origen "tradicional" y los provenientes de países que recién se han convertido en emisores de migrantes al norte de Chile. Entre los primeros están los peruanos y bolivianos y, entre los segundos, colombianos, ecuatorianos y, en general, caribeños. Estos últimos son clara minoría, pero a raíz de diversos elementos históricos son los propiamente visibilizados por los docentes como migrantes: "En este sentido, es diferente trabajar con migrantes que provienen de países más lejanos, ya que para nosotros también no son tan común, por lo que también se torna un desafío educativo contextualizar de mejor manera los contenidos, hacer una enseñanza más especializada, etc." (Profesor de CPN).

Tales distinciones aluden al carácter introvertido de los "migrantes tradicionales" y su disposición aplicada al estudio, en oposición a la extroversión de los "nuevos" migrantes: "Lo que yo valoro en ellos, sobre todo en los peruanos y bolivianos, en los colombianos no lo he visto, es un gran respeto y valoración a la escuela. Ellos creen que cometieron una cosa gravísima y a veces son cosas simples, que hay que ir corrigiendo en el camino nomás, pero esos casos son un detalle importante para ellos" (Profesor de E12). Situación que reafirma la mayoría de los docentes, como en este otro testimonio: "Se nota bastante cuando un estudiante migrante es de sectores cercanos a Chile, ya sea Perú o Bolivia. Sin embargo, se evidencia una diferencia notable con estudiantes que provienen de 
países como Colombia o países de Centroamérica, estos tienen una personalidad más extrovertida, son más 'puntudos'3 a la hora de enfrentar situaciones, quizás por el contexto social en el que viven” (Profesor de CPN).

La introversión de muchos de los migrantes de origen peruano y boliviano puede interpretarse como un mecanismo de protección debido a que han migrado a un país que históricamente los percibe como "enemigos", a raíz de la Guerra del Pacífico. Los docentes no pasan por alto este hecho: "lo que hemos descubierto es el detalle, que no sabemos si es por temor o porque algo les ocurre. Un grupo importante de ellos reniega su origen, que no sé si lo hacen porque todos se adaptan a la identidad nacional, para lograr ciertas cosas. Pero eso pasa, eso he visto yo" (Profesor de E12).

Recordemos que las personas de origen peruano y boliviano en esta zona es de larga data, más aún si se advierte que hasta 1880 esta era territorio peruano. Todo esto lleva a que los estudiantes migrantes de Perú y Bolivia experimenten una suerte de relativa invisibilidad: "Introvertidos, tratan de pasar desapercibidos" (Profesor de CCRSH), "Las diferencias que hay son mínimas, más en esta zona norte de Chile" (Profesor de LA-1opp).

En el imaginario de algunos docentes, la inclusión, expresada en una mayor socialización con su contexto de acogida, se traduce en una mayor identidad entre este y el migrante:

En algunos casos hemos notado que quieren renegar de su origen, a pesar que la escuela está acostumbrada a tener esa realidad. Al principio ellos se sienten como a la defensiva, pero con el tiempo se van dando cuenta de que como es una realidad habitual en la escuela: que al principio les cuesta socializar, por su condición de extranjero, pero con el tiempo se van identificando más con ser chilenos. [...] El único detalle importante: tenemos un nińo en octavo que es de origen colombiano, pero casi nadie sabe que es colombiano, y no le gusta hablar de Colombia. También una niñita que es boliviana, igual le molesta que le saquen el tema. Tenemos un colombiano chiquitito que está en quinto (el profesor sonríe), y a él le encanta que se sepa que viene de allá, que come esto y todo; pero esto no pasa con los más grandes (Profesor de E-12).

Como se puede apreciar, las dificultades sobre la aceptación o incomodidad en cuanto a que se sepa su origen, se evidencia más entre los estudiantes migrantes de mayor edad; esto no ocurre entre los más pequeños, quienes asumen con más naturalidad esa situación. Algunos profesores afirmaban que este hecho

3 "Puntudo" es una palabra de uso común en Chile para referirse al carácter extrovertido y a veces irreverente de algunos jóvenes. 
sería un fenómeno momentáneo y asociado al proceso de adaptación: “Debe ser la educación que ellos reciben allá, quizás los profesores son más estrictos, pero no creas que tanto tampoco, porque igual después de a poquito van agarrando confianza, entonces ya después, como se sienten igual a sus otros compañeros, ellos hacen y deshacen nomás" (Profesor de CLDV).

\section{Políticas inclusivas a nivel institucional de nińos migrantes}

En cuanto a la institucionalización de las estrategias inclusivas, los docentes reiteran el discurso respecto de que no son necesarias: "No, tal como he dicho anteriormente, se integran a tal punto que no se hace necesario. Aquí no hay, yo creo porque no hay un grupo muy grande de nińos migrantes." (Profesor de LBJNF). Y otro profesor señalaba: "No hay nada extra en el colegio aparte de ello y es porque no lo amerita el caso. No existen casos de discriminación por etnia o por nacionalidad en el colegio hasta el día de hoy y no conozco yo problemas de ese tipo. Entonces el problema no se aborda porque no se visualiza. Quizás exista, pero no se visualiza porque no hay quejas, denuncias ni nada de eso" (Profesor de CCRSH).

Sumado a lo anterior, para los docentes entrevistados, parte importante de las tareas sobre la inclusión deben atenderlas profesionales extra aula, como parte de sus funciones naturales, sin un carácter especial que se dirija a este colectivo:

Hemos apoyado migrantes, sí, buscando, por ejemplo, trabajo para alguna personas que viven en ciertas condiciones más o menos difíciles. Se entrega apoyo y asistencialidad, todo lo que se pueda apoyar para ello, pero no es algo que se haga específico con ellos, se hace con todos. Como nosotros hemos visto que ellos se han adaptado bien al sistema, no ha surgido la necesidad, porque tú instalas prácticas cuando ves algún área deficiente. Los alumnos que han llegado aquí, migrantes, se han adaptado bien al sistema, académica y conductualmente. No se hace una diferencia. El mismo de la dupla psicosocial o el comité de convivencia escolar es general para todos. Incluso en ninguna parte te sale el nombre de los alumnos y cuál es su origen, sino que la única vez que yo tengo que hacer una diferencia del origen es cuando piden este documento mensualmente, para saber cuántos migrantes tenemos. Pero lo demás es exactamente el mismo trato, porque no hemos visto dificultad de adaptación (Profesor de E-12).

Otro testimonio muestra la tendencia generalizadora entre los profesores: "Obviamente, hay algunos casos especiales que se tratan con especialistas, ya que de esos tienen que tener más cuidado, pero los casos que son de los emigrantes son todos iguales" (Profesor de CLDV). 


\section{Perfeccionamiento institucional}

La evaluación de los profesores entrevistados respecto de las posibilidades de perfeccionamiento en temas de interculturalidad y discriminación es muy divergente, pues si bien reconocen una cierta acción planificada institucionalmente, eso depende en exclusiva de su propio interés: "me las han ofrecido, pero yo no la he aceptado porque debo terminar un magíster, pero no sé si esas capacitaciones abordan el tema de la integración. Lo que sí, nos juntamos constantemente con el grupo de proyecto de integración, que ve otro tipo de exclusiones, pero también se incluye ahí al que tenga diferencias, la nacionalidad es una diferencia” (Profesor de CCRSH).

Los profesores reconocen lo mostrado por Mondaca \& Gajardo (2013), en relación a que el tratamiento de la interculturalidad se ha enfocado en los estudiantes que descienden de pueblos originarios, en particular del aimara, excluyendo otras minorías: "me invitaron a participar en un taller de lengua aimara, pero no fui [dice sin mostrar interés]" (profesor de EEE-5).

Un grupo de docentes sostenía que ello no era una necesidad, que las estrategias tendrían que ser las mismas, asumiendo un modelo de educación general para todos los estudiantes: "Nuestro establecimiento no genera instancias de capacitación debido a que son minoría y se integran bien" (Profesor de LBJNF). Aunque otros profesores indicaban que precisaban del apoyo institucional a fin de perfeccionarse para enfrentar situaciones tan básicas como los modismos:

De repente, sería bueno que tuviéramos algún apoyo, algunas guías que nos permitan ver cuál es el término en mi caso, en matemáticas, cuál es el término... los términos que usan ellos en ese país para poder decirle al alumno, no sé, sí estoy hablando de denominador, de repente ellos le tienen otro nombre... entonces, para decirles " los denominadores es lo mismo que..." le llaman así en tu país, de repente, sería más fácil que el alumno pueda entender (Profesor de NAC).

\section{Diferencias entre colegios particulares (subvencionados) y municipales}

Algunos docentes de colegios municipales afirmaban que la relación con los migrantes es distinta en función de darse en un colegio fiscal o particular subvencionado, otorgándole un rol específico a los municipales: "La integración de los migrantes es muy buena dentro de los colegios municipales. Se está haciendo muy buen trabajo en esa área" (Profesor de ED-14RR). Incluso otros consideraron que el número de estudiantes migrantes asistentes a escuelas municipales obedecería en ocasiones no solo a su mayor facilidad de acceso, sino también a una preferencia consciente: 
Nosotros, como escuela pública, no tenemos ninguna dificultad en recibir estos alumnos migrantes. Los problemas surgen en las escuelas que son particulares subvencionadas, porque a veces hay un problema de recepción de documentos. Entonces yo diría que nosotros podríamos hasta promocionar o hasta crear un sello de recibir alumnos migrantes. No hay baja de rendimiento académico y no son malos alumnos. En esta escuela son todos muy bien acogidos, [...], porque probaron otros sistemas escolares. Nosotros no hacemos una diferencia con ellos, sino que los alentamos igual que el resto. Pero si llegan de otro lado [otras escuelas de Arica], es porque ellos sintieron que la educación particular subvencionada no los acoge bien. Según la experiencia que tengo es que en la educación particular subvencionada tiene un gran sesgo con los migrantes (Profesor de EE-5).

Hay profesores que laboran en colegios particulares subvencionados y que señalan haber vivido experiencias más comprometidas con la inclusión en los municipales: "En mi anterior establecimiento educacional, que era la escuela América, había alta tasa de estudiantes migrantes y se podría decir que existía una política de integración de los niños. Se llevaba informe de estudiantes migrantes, cuando estos presentaban algún caso de interés o atención delicada" (Profesor CCRSH).

De ser una situación real, podría entenderse por la importancia que ha adquirido en los últimos tiempos la matrícula de estudiantes migrantes para la supervivencia de los establecimientos municipales debido a la fuga de estudiantes chilenos al sector particular subvencionado. Sin embargo, dichas diferencias no son detectadas ni por apoderados ni por estudiantes migrantes. Las situaciones asociadas a las complejidades de adaptación se relacionan sobre todo con el tiempo que los estudiantes llevan como migrantes en el país de acogida. En relación con esto, al menos en la muestra seleccionada para esta investigación, los estudiantes migrantes con más tiempo en el país y los que no tuvieron en Arica el primer destino de llegada estudian en colegios particulares subvencionados. Por ende, los que tienen menos tiempo en Chile Arica sería su primer destino de llegada y la opción municipal el primer contexto educativo en el país de acogida.

\section{Conclusiones}

La emergencia de la realidad migrante en las escuelas chilenas de frontera desafía los objetivos y estrategias de políticas públicas, en particular al modelo de gestión de la diversidad que se observa en las representaciones de la práctica docente y del proceso de inclusión en general. 
Las representaciones que de los profesores se han desglosado en este artículo evidencian lo que se ha llamado "sociedad normalizada", en la cual se eliminan las diferencias mediante estructuras y procedimientos que uniforman la realidad escolar. Los nudos críticos en el desarrollo de este proceso son interpretados desde la causalidad, donde las problemáticas de la familia migrante fungen como obstáculos al éxito académico.

El modelo de gestión de la diversidad cultural dominante en dichas representaciones transita entre el multiculturalista, que acepta las diferencias pero que no acaba con las jerarquías emergentes de la asimetría que caracteriza al proceso migratorio, y el asimilacionista, que enfrenta la tensión de la pertenencia sin gestionar cambios en los procesos educativos, y en el que existe el convencimiento de que el estudiante debe "hacer méritos" y adaptarse al sistema escolar.

La inclusión como estrategia para eliminar las barreras para el el éxito académico aparece interpretada de modo incipiente, pues se sigue visualizando la diferencia como un problema que se debe eliminar, dada la minoría que todavía son los estudiantes migrantes. Esto revela las tareas normalizadoras, generalmente protagonizadas por la escuela pública como el agente más importante. Así, los buenos resultados en calificaciones y disciplina que presenta la mayor parte de los estudiantes migrantes se transforma en el principal argumento para el statu quo. Es probable que la falta de estrategias a nivel institucional que promuevan la inclusión genera una recursividad alimentando y promoviendo la permanencia del modelo descrito.

En el discurso de los docentes, el concepto de "normalidad" se usa habitualmente para indicar que la diferencia es un problema individual, identificando el enfoque "biomédico", minimizando el rol que el contexto de acogida tiene en el proceso.

El desinterés de los profesores por conocer el contexto social en el que desempeña su labor, los lleva a que no se cuestionen acerca de la situación de los estudiantes migrantes y a dejar que estos sean quienes asuman - muchas veces en solitario - la lucha por la inclusión. Una tarea a seguir sería evaluar si esta falta de problematización no se debe precisamente a la ausencia de estrategias institucionales que visibilicen esa situación y a que no se comprende la complejidad de la diversidad cultural en el sistema educativo.

Asimismo, no debe perderse de vista que la evaluación de la inclusión se asocia a las calificaciones y conductas de los estudiantes migrantes, considerándolos exitosos cuando no generan "problemas", pero obviando las conductas de los estudiantes nacionales como factor relevante de este proceso.

El carácter fronterizo de Arica y su relación histórica con Perú y Bolivia marcan la representación del proceso de inclusión en la zona, pero invisibilizan al migrante tradicional. 
Sigue siendo un tema pendiente la sensación expresada por los docentes respecto a que el éxito de la incorporación de los estudiantes migrantes se traduce en la mayor homogeneización alcanzada por ellos en la sociedad de acogida.

\section{Referencias}

Abajo, J. \& Carrasco, S. (2004). Experiencias y trayectorias de éxito escolar de gitanas y gitanos en España. Encrucijadas sobre educación, género y cambio cultural. Madrid: CIDE/Instituto de la Mujer. Recuperado el 14 de octubre de 2015, de https://www.gitanos.org/upload/04/70/1.5 -EXP_Experiencias_y_trayectorias_de_exito_escolar_de_gitanas_y_gitanos_en_Espana.pdf

Alvites, L. \& Jiménez, R. (2011). Nińos y niñas migrantes, desafío pendiente. Innovación educativa en escuela de Santiago de Chile. Synergies Chili, (7), 121-136.

Booth, T. \& Ainscow, M. (2002). Indice de inclusión: desarrollando el aprendizaje y la participación en las escuelas. Santiago de Chile: UNESCO-OREALC.

Caballero, Z. (2001). Aulas de colores y sueños: la cotidianeidad en las escuelas multiculturales. Barcelona: Octaedro.

Castro, A. (2011). Acculturation Strategies and Psychological and Sociocultural Adaptation of Foreign Students in Argentina. Interdisciplinaria, 28(1), 115-130.

Díaz, A., Ruz, R., Galdames, L. \& Tapia, A. (2012). El Arica peruano de ayer. Siglo xix. Atenea, (505), 159-184.

Dovigo, F. (2014). El tratamiento de la diversidad en las instituciones educativas. En Gairín, J. (Coord.). Colectivos vulnerables en la Universidad. Reflexiones y propuestas para la intervención (pp. 87-118). Madrid: Wolters Kluwer España.

Duschatzky, S. \& Skliar, C. (2000). La diversidad bajo sospecha. Reflexiones sobre los discursos de la diversidad y sus implicancias educativas. Cuaderno de Pedagogía Rosario, IV(7), 1-13.

Gairín, J. \& Iglesias, E. (2010). El programa curricular en contextos escolares con fuerte presencia de los alumnos de familia inmigrante. Bordón, 62(1), 61-75.

Gairín, J. (Coord.). (2014). Colectivos vulnerables en la Universidad. Reflexiones y propuestas para la intervención. Madrid: Wolters Kluwer Educación. Recuperado el 16 de octubre de 2015, de https://www.dropbox.com/s/7jb4abuoqpyyloq/ACCEDES\%20II_2014.pdf?dl=0

Giovine, R. \& Martignoni, L. (2011). La escuela media bajo el mandato de la obligatoriedad, Cadernos Cedes, 31(84), 175-194. 
Glaser, B. \& Strauss, A. (1967). Discovery of Grounded Theory. Chicago: Aldine.

Gómez, J. (2005). Pautas y estrategias para entender y atender la diversidad en el aula. Pulso, (28), 199-214.

González, S. (2002). Chilenizando a Tunupa. La escuela pública en el Tarapacá andino 1880-1990. Santiago: DIBAM.

Hannoun, H. (1992). Els guettos de l'escola. Per una educació intercultural. Vic: Eumo Editorial.

Hernández, M. (2011). La migración peruana en Chile y su influencia en la relación bilateral durante el gobierno de Michelle Bachelet (2006-2010). Tesis de Maestría en Estudios Internacionales. Instituto de Estudios Internacionales-Universidad de Chile. Santiago de Chile.

Hombrados, I. \& Castro, M. (2013). Apoyo social, clima social y percepción de conflictos en un contexto educativo intercultural. Anales de Psicología, 29(1), 108-122. Recuperado el 21 de octubre de 2015, de http://dx.doi.org/10.6018/analesps.29.1.123311

Imilán, W., Márquez, F. \& Stefoni, C. (2015). Rutas migrantes en Chile. Habitar, festejar y trabajar. Santiago de Chile: Ediciones Alberto Hurtado.

Jackson, P. (2001). La vida en las aulas. Madrid: Morata.

Jiménez, F. (2013). Discursos y prácticas educativas en la escuela multicultural. Tesis doctoral. Universidad Autónoma de Barcelona.

Jiménez, L. (2007). Cuando estudiamos pertenencias étnicas en educación ¿nos ayudan los modelos de análisis basados en categorías étnicas? EMIGRA Working Papers, 102. Recuperado el $22 \mathrm{de}$ julio de 2014, de www.emigra.org.es.

Martín-Crespo, Ma . \& Salamanca, A. (2007). El muestreo en la investigación cualitativa. Nure Investigación, (27). Recuperado el 14 de octubre de 2015, de http://www.nureinvestigacion .es/OJS/index.php/nure/article/view/340/330

Mendoza, R. (2014). Evaluando políticas públicas educativas etnográficamente: reflexiones metodológicas. En Cárcamo Vásquez, E. (Ed.). Contextos múltiples de socialización y aprendizaje. Un análisis desde la etnografía de la educación. Making of... Construcciones etnográficas de la educación. Recuperado el 14 de octubre de 2015, de http://e-spacio.uned.es/fez/eserv/ bibliuned:500383-IIICongresoEtnografia-1005/Documento.pdf

Mondaca, C. (2008). Identidades sociales y representaciones políticas en conflicto: El sistema educativo chileno en Los Andes de Arica, 1884-1929. Revista Anthropológica, 26(26), $33-62$. 
Mondaca, C. \& Aguirre, C. (2011). Estado nacional y comunidad andina. Disciplinamiento y articulación social en Arica, 1880-1929. Revista de Historia, 44(1), 5-50.

Mondaca, C. \& Gajardo, Y. (2013). La educación intercultural bilingüe en la región de Arica y Parinacota, 1980-2010, Diálogo Andino, (42), 69-87. Recuperado el 4 de octubre de 2015, de http://www.scielo.cl/scielo.php?script=sci_arttext\&pid=S0719-26812013000200007\&lng =es\&tlng=es. 10.4067/S0719-26812013000200007

Mondaca, C., Rivera, P. \& Aguirre, C. (2013). La escuela y la Guerra del Pacífico. Propuesta didáctica de historia para la inclusión educativa en contextos transfronterizos del norte de Chile. Si Somos Americanos. Revista de Estudios Transfronterizos, 13(1), 123-148.

Mondaca, C., Gajardo, Y. \& Sánchez, E. (2014). Violencia sociopolítica en Arica y Tacna, 19001920. En Díaz Araya, A., Ruz Zagal, R. \& Galdames Rosas, L. (Comps.). Tiempos Violentos. Fragmentos de Historia Social en Arica (pp. 63-74). Arica: Ediciones Universidad de Tarapacá.

Mondaca, C., Rivera, P. \& Gajardo, Y. (2014). Educación parvularia e inclusión en el norte de Chile. Formando pequeños chilenos en las aulas de Tarapacá. Revista Alpha, (39), 251-266.

Morales, V. \& Molina, C. (2003). Reasentamiento involuntario: integración y civilización. Bitácora Urbano Territorial, 1(7), 19-25. Recuperado el 3 de octubre de 2015, de http://www .redalyc.org/articulo.oa?id=74810704

Morín, E. (1995). El pensamiento complejo. Madrid: Gedisa.

Navas, L., Holgado, F. \& Sánchez, A. (2009). Predicción de los estereotipos académicos ante los estudiantes inmigrantes. Horizontes Educacionales, 14(2), 37-47.

Pavez Soto, I. (2013). La infancia como sujeto de las politicas públicas e intervenciones sociales: El caso de la niñez migrante en el Chile del siglo XXI. Recuperado el 3 de octubre de 2015, de http:// actacientifica.servicioit.cl/biblioteca/gt/GT9/GT9_PavezSotoI.pdf

Poveda, D. (2003). Saberes sociolingüísticos en una clase multicultural. En Poveda, D. (Coord.). Entre la diferencia y el conflicto. Miradas etnográficas a la diversidad cultural en educación (pp. 67-98). Cuenca: Ediciones de la Universidad de Castilla-La Mancha.

Romero, J. (2004). Latinoamérica: Las ciudades y las ideas (2a. ed.). Buenos Aires: Siglo XXI Editores.

Riquelme, J. \& Alarcón, G. (2008). El peso de la historia en la inmigración peruana en Chile. Revista Polis, 7(20), 299-310.

Sauquillo, J. (1987). Poder político y sociedad normalizada en Michael Foucault. Revista de Estudios Políticos (Nueva Época), (56), 181-203. 
Schmelkes, S. (2009). Interculturalidad, democracia y formación valoral en México. Revista Electrónica de Investigación Educativa, 11(2), 1-10.

Taylor, S., Sherman, D., Kim, H., Jarcho, J., Takagi, K. \& Dunagan, M. (2004). Culture and Social Support: Who Seeks it and Why? Journal of Personality and Social Psychology, (87), 354-362.

Tarrow, N. (1990). A Tri-level Model of Intercultural Education in Two Regions of Spain. Frankfurt: Deutsches Institut für Internationale Pädagogische Forschung.

Tolosana, C. (2014). La atención a grupos vulnerables. Un reto social y universitario. En Gairín, J. (Coord.). Colectivos vulnerables en la Universidad. Reflexiones y propuestas para la intervención (pp. 21-31). Madrid, Wolters Kluwer España.

Touraine, A. (2000). ¿Podremos vivir juntos? Iguales y diferentes. México: Fondo de Cultura Económica.

Valdivieso, P. (2007). A propósito de las relaciones Chile-Bolivia-Perú: Percepciones, experiencias y propuestas. Bicentenario. Revista de Historia de Chile y América, 6(2), 99-123.

Vásquez, A. \& Martínez, I. (1996). La socialización en la escuela. Una perspectiva etnográfica. Barcelona: Paidós.

Woods, P. (1987). La escuela por dentro. La etnografia en la investigación educativa. Barcelona: Paidós.

Zapata, A. (2011). Vida familiar en el contexto de la migración internacional materna o paterna. Hijos e hijas que reciben remesas. Medellín: Comfenalco.

Recibido el 13 de octubre de 2015. Aceptado el 12 de diciembre de 2016. 